\title{
CD4 T Cells Treated with gp120 Acquire a CD45R0+/CD45RA+ Phenotype
}

\author{
Sergey A. Trushin ${ }^{1,2}$, Gary D. Bren ${ }^{1}$, Andrew D. Badley ${ }^{* 1,2}$ \\ ${ }^{I}$ Division of Infectious Diseases, ${ }^{2}$ Program in Translational Immunovirology and Biodefense, Mayo Clinic, Rochester, \\ MN 55905, USA
}

\begin{abstract}
HIV-infected patients exhibit quantitative and qualitative defects in CD4 T cells, including having increased numbers of $\mathrm{CD} 4+\mathrm{CD} 45 \mathrm{R} 0+/ \mathrm{CD} 45 \mathrm{RA}+\mathrm{T}$ cells, although it remains unclear how these cells arise. Here we demonstrate that gp120 treatment of activated but not resting primary human CD4 T cells decreases number of cells with single positive CD45R0+/CD45RA- effector memory phenotype while proportionally increasing the subset of cells with double positive CD45R0+/CD45RA+ mixed phenotype. We found that double positive CD45R0+/CD45RA+CD4 T cells preferentially undergo apoptosis while single positive CD45R0+/CD45RA- and CD45R0-/CD45RA+ do not. Blocking gp120CD4 interaction with sCD4 or inhibition Lck activity reverses gp120 induced increase in double positive CD45R0+/CD45RA+CD4 $\mathrm{T}$ cells and subsequently diminishes the apoptosis of double positive CD45R0+/CD45RA+ cells. Altogether these data indicate that gp120 ligation of the CD4 receptor increases the number of double positive $\mathrm{CD} 45 \mathrm{R} 0+/ \mathrm{CD} 45 \mathrm{RA}+\mathrm{CD} 4 \mathrm{~T}$ cells which subsequently undergo apoptosis in a CD4 dependent manner.
\end{abstract}

\section{INTRODUCTION}

One hallmark of HIV pathogenesis is a decline of CD4 T cell number that results from the death of both HIV-1 infected CD4 T cells, as well as uninfected cells. Both HIV expressed proteins, as well as immune activation, contribute to this $\mathrm{T}$ cell death [1]. Immune activation leads to an increase in $\mathrm{T}$ cell turnover from enhanced proliferation which in turn is associated with high rates of apoptosis $[2,3]$. The magnitude of immune activation correlates with the level of HIV viremia [4]. Increased expression of immune activation markers (HLA-DR+, CD38+, CD45RO+, and CD95) also correlates with higher apoptosis rates of CD4 T cells [5]. Importantly, suppression of viral replication with HAART treatment reduces immune activation [6,7], normalizes expression of activation markers, and decreases CD4 $\mathrm{T}$ cell apoptosis [8-10].

As a result of chronic immune activation, central memory and naïve CD4 T cells are constantly recruited into the effector pool $[11,12]$ resulting in dramatic changes in populations of naïve, effector and central memory CD4 T cells [13]. The mechanisms of how the ratio of these CD4 T cells is altered during HIV infection are not completely understood.

In particular, chronic HIV infection results in an increased number of $\mathrm{CD} 4 \mathrm{~T}$ cells with a peculiar double positive $\mathrm{CD} 45 \mathrm{RO}+/ \mathrm{CD} 45 \mathrm{RA}+/ \mathrm{CD} 25+\mathrm{CD} 4+$ phenotype, and this subset is further increased following intermittent IL-2 therapy [14]. The origin of this subset was previously ascribed to be transition phase of naïve CD45RO-/CD45RA+CD4 T cells transitioning to a memory CD45RO+/CD45RA-CD4 T cell phenotype [15]. Furthermore, HAART with IL-2 therapy selectively increases the number of activated CD4 T cell expressing CD45RO+/CD25+ [16]. Here we describe a

*Address correspondence to this author at the Division of Infectious Diseases, Mayo Clinic, 200 First Street SW, Rochester, MN 55905, USA; Tel: 507-266-5065; Fax: 507-284-3757; E-mail: badley.andrew@mayo.edu novel effect of HIV-1 gp120 signaling through CD4 resulting in altered CD45 isoform expression by CD4 T cells.

\section{MATERIALS AND METHODOLOGY}

\section{Cell Culture and Reagents}

This protocol was reviewed and approved by the Mayo institutional review board. CD4+ T cells were isolated from the blood of healthy volunteer blood donors by using RossetteSep CD4 enrichment cocktail (StemCell Technologies, Vancouver, British Columbia, Canada), producing 98\% pure CD4+ T cells as determined by flow cytometry. CD4 T cells were maintained in RPMI 1640 supplemented with $10 \%$ fetal bovine serum (Invitrogen, Carlsbad, CA), $2 \mathrm{mM}$ Lglutamine, and antibiotics (penicillin $100 \mathrm{U} / \mathrm{ml}$, streptomycin $100 \mu \mathrm{g} / \mathrm{ml})$ at $0.5 \times 10^{6}$ cells $/ \mathrm{ml}$. CD4+ T cells used in the various experiments were stimulated with PHA $(1 \mu \mathrm{g} / \mathrm{ml})$ for 24 hours, and then cells were washed twice with RPMI 1640 and maintained in media supplemented with $50 \mathrm{U} / \mathrm{ml}$ of IL-2 for 48-72 hours. CD4 T cells were incubated with HIV-1 X4 gp120IIIB (Immuno Diagnostics, Inc. Woburn, MA) or gp120 IIIB pretreated with soluble CD4 (1:2 ratio) (Immuno Diagnostics, Inc. Woburn, MA) at concentrations of 1 $\mu \mathrm{g} / \mathrm{ml} / 2 \times 10^{6}$ cells for 24 hours at $37^{\circ} \mathrm{C}$. AMD3100 (NIH AIDS Research and Reference program) was used at $2 \mu \mathrm{M}$ for 30 minutes at $37^{\circ} \mathrm{C}$. PP2 was purchased from CalBiochem (La Jolla, CA). Anti-CD4-PE, anti-CD25-FITC, antiCD69-PE, anti-CD4PerCP, anti-CD62L-PE, anti-CD45ROFITC, anti-CD45RA-PE-Cy-7, anti-HLA-DR-PE, AnnexinV-Cy-5, AnnexinV-APC, IgG1к-PE-Cy7, IgG2a-FITC, $\operatorname{IgG} 1 \kappa-\mathrm{PE}$ and propidium iodine were purchased from BD Biosciences (San Jose, CA).

\section{Cell Death Analysis and Flow Cytometry}

CD4 T cells were untreated or pre-incubated with specific inhibitors and stimulated with either BSA or with soluble gp120IIIB $(10 \mu \mathrm{g} / \mathrm{ml})$ overnight. The following day, cell death was analyzed by staining with AnnexinV-Cy-5 and propidium iodine following the manufacturer's instructions 
from BD Biosciences (San Jose, CA). All experiments were performed at least three times.

$\mathrm{T}$ cell phenotyping studies were performed by using fourcolor Flow analysis on FACSCanto cytometer and using FACSDiva 6.0 software. Briefly, $2 \times 10^{6}$ cells were resuspended in $200 \mu 1$ of PBS $+0.5 \%$ BSA, stained with the indicated primary conjugated antibodies for 20 minutes (antiCD45RO-FITC, anti-CD45RA-PE-Cy-7, anti-CD27 APC and anti-CD62L-PE), washed, fixed and then analyzed. In some cases, for analysis of cell death, cells were stained in binding buffer $(140 \mathrm{mM} \mathrm{NaCl}, 10 \mathrm{mM}$ HEPES/NaOH $(\mathrm{pH}$ 7.4), $2.5 \mathrm{mM} \mathrm{CaCl} 2)$ as described above except that antiCD62L-PE was substituted with AnnexinV-PE.

\section{RESULTS}

Gp120 Decreases CD45RO+ Memory Phenotype in Activated But Not Resting CD4 $T$ cells

We and others have previously demonstrated that gp 120 induces death of resting CD4 $\mathrm{T}$ cell in a CXCR4-p38 dependent manner [17]. However, the effect of gp120 on activated CD4 $\mathrm{T}$ cells is largely unknown. To examine the effect of gp120, resting (CD4+CD25-CD69-HLA-DR-) and activated (CD4+ CD25+CD69+HLA-DR+) primary human CD4 $\mathrm{T}$ cells were treated with gp120 IIIB $(10 \mu \mathrm{g} / \mathrm{ml})$ for 24 hours and then CD4 $\mathrm{T}$ cells were analyzed for CD45R0 and CD45RA expression by flow cytometry. As shown in Fig. (1A), gp120 treatment results in a $50 \%$ decrease in the subset of single positive CD45R0+/CD45RA- $(31.85 \pm 0.63 \%$ with
BSA, $13.80 \pm 3.73 \%$ with gp120, $p=0.01$ ) memory cells when activated but not resting CD4 T cells are treated with gp120 (34.25 $\pm 1.1 \%$ with BSA, $28.95 \pm 1.96 \%$ with gp 120 , $\mathrm{p}=0.08$ ). In parallel, there is a proportional increase in the subset of double positive CD45R0+/CD45RA+ mixed phenotype CD4+ T cells (Fig. 1B) as well as an increase in the proportion of Annexin-V positive CD4 T cells (Fig. 1C). While the increased apoptosis of resting cells following gp120 is due to CXCR4 signaling [17], the mechanisms by which gp120 causes death in activated cells are unknown.

The finding that gp120 treatment of activated cells increases the lymphoid homing marker, CD27 (13.8\% of total CD4 $\mathrm{T}$ cells) preferentially in double positive CD45R0+/CD45RA+ cells suggests that these cells might be the specific cell type which dies following gp120 treatment of activated CD4 T cells (Fig. 2A, B). We therefore measured absolute number and proportion of Annexin-V positivity of double positive CD45R0+/CD45RA+, single positive CD45R0-/CD45RA+ and CD45R0+/CD45RA- subsets by using four color flow cytometry analysis. First we observed that CD45RO+RA- proportion decreased $(39.42 \pm 3.44 \%$ with BSA, $27.31 \pm 6.38 \%$ with gp 120 ) while CD $45 \mathrm{RO}+\mathrm{RA}+$ population increased $(35.97 \pm 2.70 \%$ with BSA, $49.42 \pm 10.05 \%$ with gp120), suggesting that the former transitioned to the latter (Fig. 3A). Next we observed that only double positive CD45R0+/CD45RA+ CD4 $\mathrm{T}$ cell subset contain a significant proportion of Annexin positive cells $(\sim 40 \%)$ in contrast to single positive CD45R0+/CD45RA- and CD45R0-/CD45RA+ subsets that do not (Fig. 3B). Therefore, gp120 signaling both

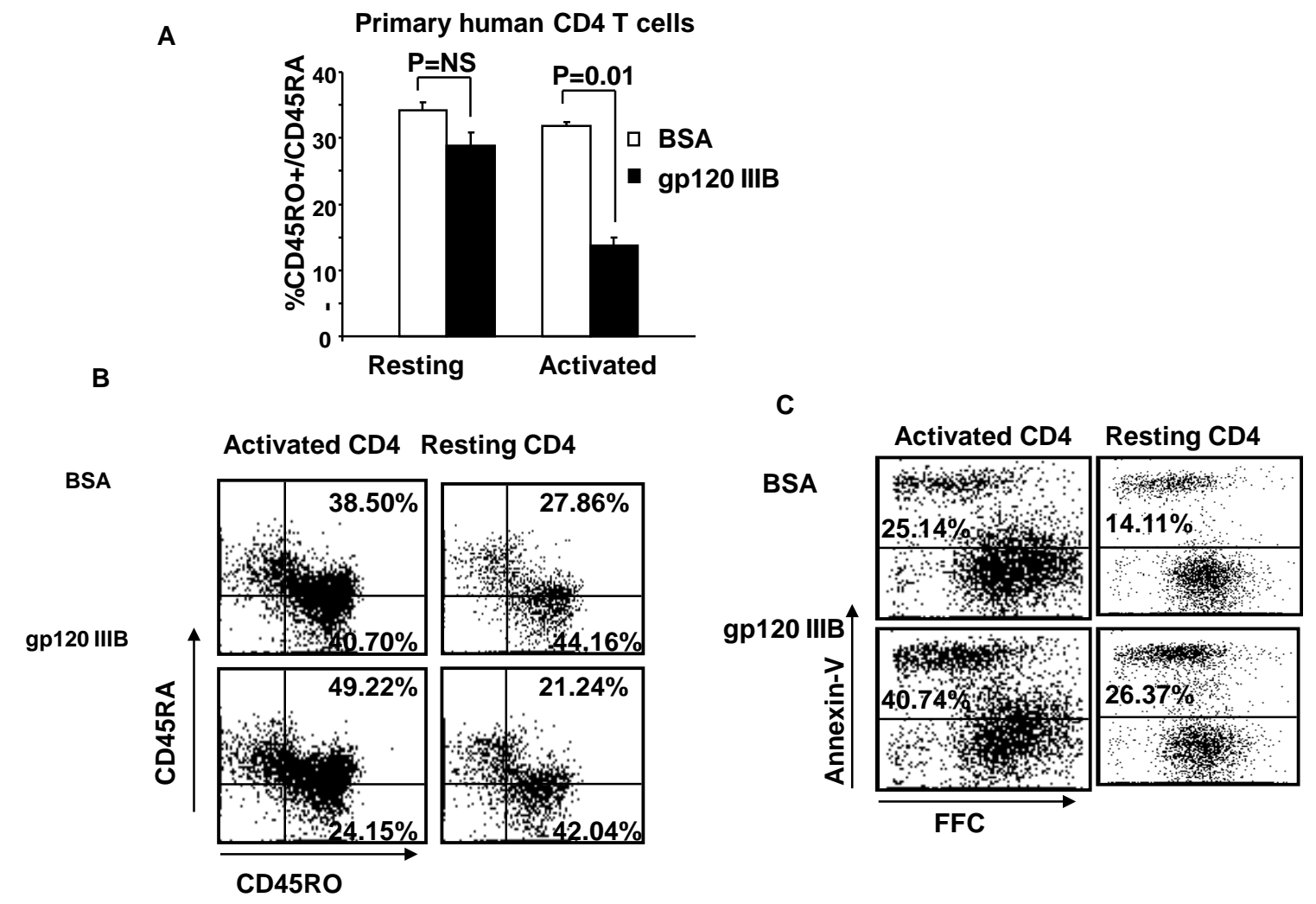

Fig. (1). Gp120 decreases CD45RO+/CD45RA- memory phenotype in activated but not resting primary human CD4 T cells. (A and B) CD4+ T cells (98\% pure) were stimulated with PHA for 24 hours or left rested, and treated with either p120 (IIIB) (1 $\mu \mathrm{g} / \mathrm{ml})$ or BSA control $(1 \mu \mathrm{g} / \mathrm{ml})$ and analyzed for CD45RO and CD45RA (gated on CD4 population) by flow cytometry. (C) Resting and activated primary human CD4 T cells were treated with gp120 (IIIB) as above and analyzed for Annexin-V positive cells. Data is representative of three independent experiments. 
activates cells towards a double positive phenotype and subsequently promotes their apoptotic death. Of interest, these Annexin positive CD45R0+/CD45 RA+ cells are also CD27+. Altogether these results indicate that gp120 treatment of activated CD4 $\mathrm{T}$ cells result in decrease memory CD45R0+/CD45RA- CD4 $T$ cells due to accumulation of CD45R0+/CD45RA + /CD27+ T cell subset that subsequently and selectively undergo apoptosis.

A

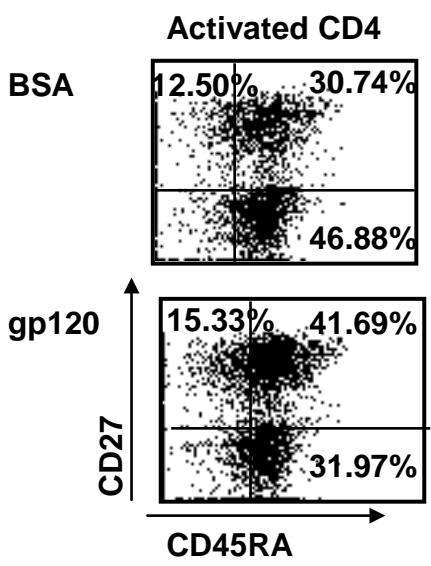

B

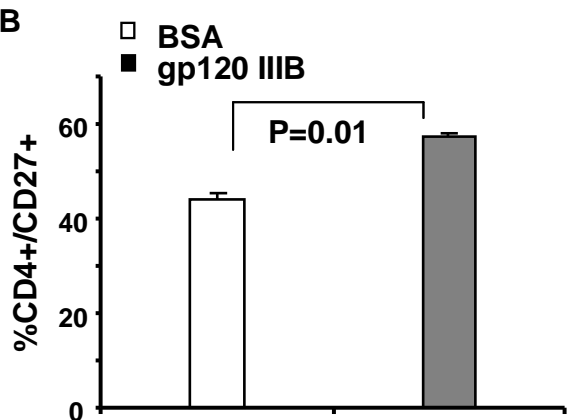

Fig. (2). Gp120 increases subset of CD27+/CD45RO+/CD4+ T cells. (A) Activated primary human CD4 T cells (98\% pure) were treated with gp120 IIIIB and the expression of CD27 and CD45RA were analyzed by flow cytometry. (B) Pooled data from three independent experiments. $\mathrm{P}$ values were determined by Student's paired t test.

Gp120 Stimulated Accumulation of CD45RO+/CD45 RA+ CD4 T Cells Requires CD4 and Lck But Not CXCR4

To investigate whether gp120 effect on CD45R0+/CD45RA+ CD4 $\mathrm{T}$ cell is due to ligation to CD4 or CXCR4, we used either soluble CD4 or the CXCR4 inhibitor, AMD3100. Independent experiments verified the activity of AMD3100 by blocking SDF-induced chemotaxis (data not shown). As shown in Fig. (4A, B), inhibition of CD4 ligation by sCD4 [17] blocks the increase in double positive CD45R0+/CD45RA+ memory cells. Consistent with this observation, inhibition of Lck activity with PP2 inhibitor also blocks increase in CD45R0+/CD45RA+ CD4 $\mathrm{T}$ cell subset. Conversely inhibition of gp120-CXCR4 interaction with AMD3100 [17] does not block completely gp120 mediated increase of CD45R0+/CD45RA+ CD4 T cells. Therefore, our results demonstrate that gp120 ligation to CD4 and subsequent activation of Lck results in accumulation of dou- ble positive CD45R0+/CD45RA+ memory CD4 $\mathrm{T}$ cells which subsequently and selectively undergo apoptosis in a CD4 dependent and CXCR4 independent manner.

\section{DISCUSSION}

In the current report we have shown that gp120 treatment of activated CD4 $\mathrm{T}$ cells decreases the subset of single positive CD45R0+/CD45RA- CD4 T cells and increases the subset of double positive CD45R0+/CD45RA+ CD4 T cells which selectively undergo apoptosis. These results imply the gp120 may play a role in depletion of memory CD45R0+/ CD45RA- CD4 T during HIV.

Previously, the CD45R0+/CD45RA+ subset of CD4 T cells was characterized as a transition from naïve to effector phenotype during CD4 priming [15, 18] with increased expression of activation markers [19, 20]. The generation of this subset can occur in two ways: (i) the transition of recently activated of CD45RA $+\mathrm{T}$ naïve cells to CD45R0+/ CD45RA+ phenotype $[15,21]$ and; (ii) re-expression of CD45RA isoform in the absence of Ag stimulation [15]. The fact that only few CD45R0+/CD45RA+ cells express markers of recently activated cells $[19,20]$ and upregulation of $\mathrm{CD} 45 \mathrm{RA}+$ (up to $10 \%$ ) by CD45RO+/CD45RA- cells [15] suggest that gp120 rather upregulates CD45RA+ on single positive CD45RO+/CD45RA- memory CD4 $\mathrm{T}$ cells rather than promoting CD45RO expression on naïve CD4 T cells. Indeed, increase of double positive CD45RO+/CD45 RA+ CD4 $\mathrm{T}$ cells is followed by proportional decrease in CD45RO+/CD45RA- memory but not decrease in naïve CD45RO-/CD45RA+ CD4 T cells.

Others have shown that PBMC stimulated with staphylococcal enterotoxin A (SEA) in the presence of V3-derived gp120 peptides results in increase of $\mathrm{CCR} 5+/ \mathrm{CXCR} 4+/$ CD45RO+ CD4 $\mathrm{T}$ cells followed by increased levels of apoptosis [22]. Our findings are in good agreement with this observation: first, both results demonstrate the gp120 mediated increase in transient state from naïve to activated CD4 T cells; second, both studies observe the increased apoptosis of activated CD4 T cells in the presence of gp120 or V3derived peptide. However, our results highlight that gp120 priming of previously activated CD4 $\mathrm{T}$ cells reverses single positive CD45RO+/CD45RA- effector phenotype to double positive CD45RO+ /CD45RA+ transient phenotype.

The observation that double positive CD45R0+/CD45 $\mathrm{RA}+$ preferentially undergo apoptosis presents one potential scenario of how inappropriately activated CD4 $\mathrm{T}$ cells die during HIV disease, and why IL-2 augments this process [16]. It is of further interest that gp120 treatment results in apoptosis of CD27+/CD45R0+/CD45RA+ cells. In fact, CD27 expression is rapidly upregulated following TCR stimulation [23] and CD27 signaling is essential for survival of Ag-primed CD4 T cells [24]. Therefore, either the lack of antigen specific signaling or the lack of CD27 signaling may explain the increased apoptosis of double positive CD45R0+/CD45RA+ following gp120 treatment. Finally, the observation that CD4 ligation by gp120 and subsequent activation of Lck is required for generation of double positive CD45R0+/CD45RA + CD4 T cells is of relevance and suggests that inhibitors of CD4-gp120 interactions may reverse the expansion of double positive CD45R0+/CD45RA+ population and subsequent increased apoptosis during HIV infection. 


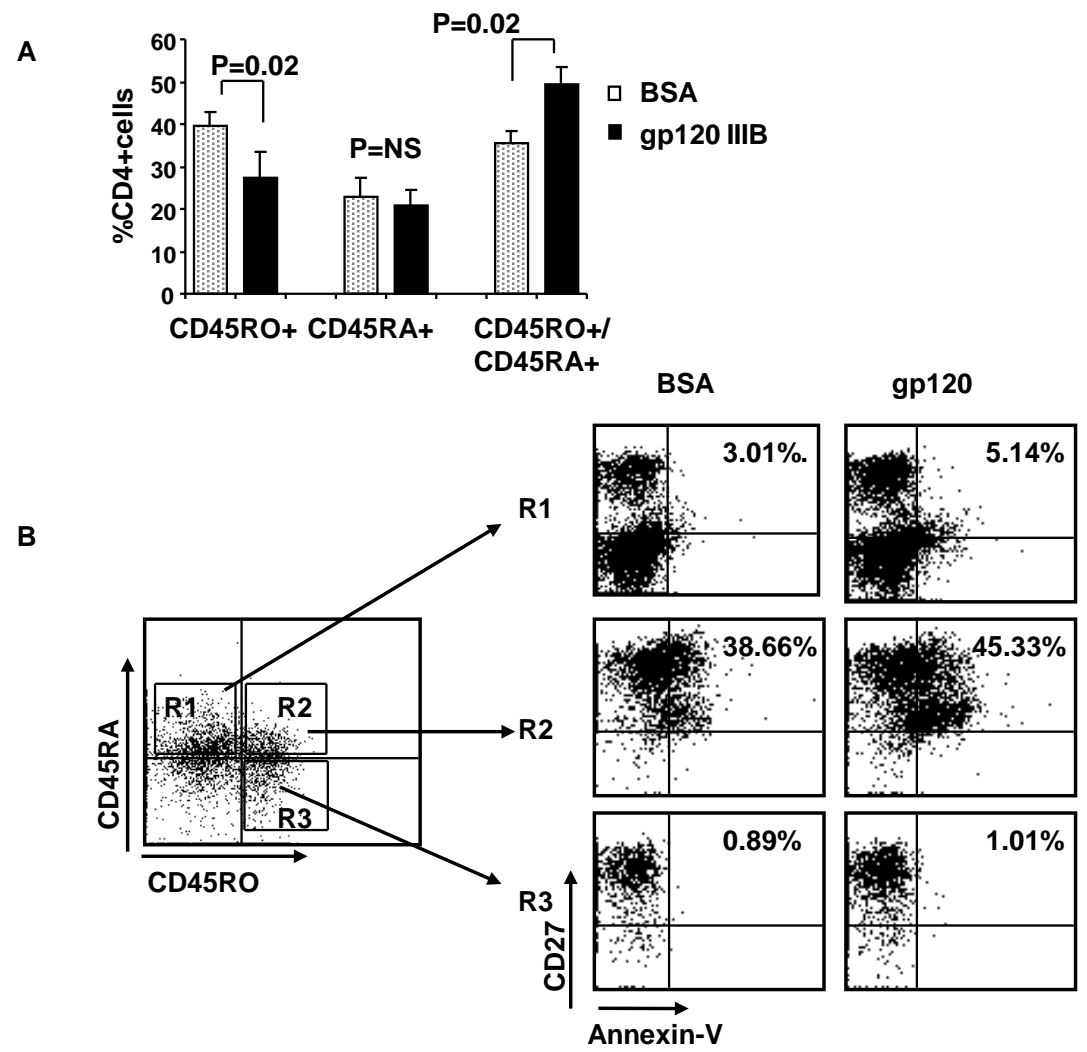

Fig. (3). Gp120 increases subset of double positive CD45RA+/CD45RO+ CD4 $\mathrm{T}$ cells that undergo apoptosis. (A) Activated primary human $\mathrm{CD} 4 \mathrm{~T}$ cells were treated with gp120 IIIIB and CD45RA+/CD45RO-, CD45RA-/CD45RO+ and double positive CD45RA+/CD45RO+ cells were quantified by flow cytometry. (B) Same as Fig. (3A) except that CD27 expression and Annexin-V positivity were measured by four color flow cytometry. The data is representative of three independent experiments.
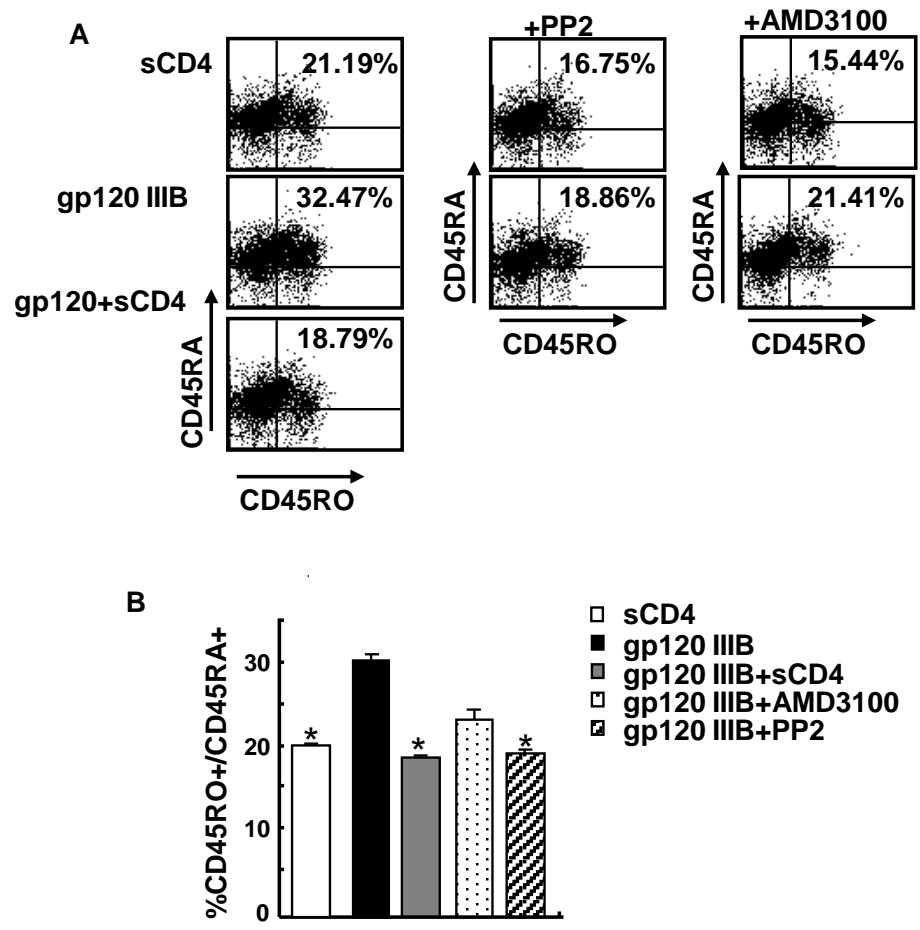

Fig. (4). CD4 and Lck are required for gp120 mediated increase of double positive CD45RA+/CD45RO+ CD4 T cells. (A) Activated primary human CD4 T cells were left untreated or were pre-treated with either Lck inhibitor, PP2 $(5 \mu \mathrm{M})$ or CXCR4 inhibitor, AMD3100 $(2 \mu \mathrm{M})$ or sCD4 $(2 \mu \mathrm{g} / \mathrm{ml})$ followed by gp120. Then number of single positive CD45RA+/CD45RO-, CD45RA-/CD45RO+ and double positive CD45RA+/CD45RO+ cells were analyzed by flow cytometry. (B) The data represents three independent experiments as described above. $\mathrm{P}$ values were determined by Student's paired $\mathrm{t}$ test. $*=\mathrm{P}<0.05$ compared to gp 120 alone. 


\section{ACKNOWLEDGEMENTS}

Grant Support: Dr. Andrew Badley is supported by the following grants: NIH R01 AI62261, R01 AI40384 and a Burroughs Wellcome Award ID \#1005160.

\section{REFERENCES}

[1] Lum J, Badley AD. In: Badley AD, Ed. Cell Death During HIV Infection. Boca Raton: Taylor and Francis Press 2006; pp. 109-126.

[2] Douek DC, Betts MR, Hill BJ, et al. Evidence for increased T cell turnover and decreased thymic output in HIV infection. J Immunol 2001; 167: 6663-8.

[3] Hellerstein M, Hanley MB, Cesar D, et al. Directly measured kinetics of circulating $\mathrm{T}$ lymphocytes in normal and HIV-1-infected humans. Nat Med 1999; 5: 83-9.

[4] Perelson AS, Neumann AU, Markowitz M, Leonard JM, Ho DD. HIV-1 dynamics in vivo: virion clearance rate, infected cell lifespan, and viral generation time. Science 1996; 271: 1582-6.

[5] Gougeon ML, Lecoeur H, Dulioust A, et al. Programmed cell death in peripheral lymphocytes from HIV-infected persons: increased susceptibility to apoptosis of CD4 and CD8 T cells correlates with lymphocyte activation and with disease progression. J Immunol 1996; 156: 3509-20.

[6] Hazenberg MD, Stuart JW, Otto SA, et al. T-cell division in human immunodeficiency virus (HIV)-1 infection is mainly due to immune activation: a longitudinal analysis in patients before and during highly active antiretroviral therapy (HAART). Blood 2000; 95: 249-55.

[7] Lempicki RA, Kovacs JA, Baseler MW, et al. Impact of HIV-1 infection and highly active antiretroviral therapy on the kinetics of CD4+ and CD8+ T cell turnover in HIV-infected patients. Proc Natl Acad Sci USA 2000; 97: 13778-83.

[8] Badley AD, Dockrell DH, Algeciras A, et al. In vivo analysis of Fas/FasL interactions in HIV-infected patients. J Clin Invest 1998; 102: 79-87.

[9] Bohler T, Walcher J, Holzl-Wenig G, et al. Early effects of antiretroviral combination therapy on activation, apoptosis and regeneration of T cells in HIV-1-infected children and adolescents. AIDS 1999; 13: 779-89.

[10] Giorgi JV, Majchrowicz MA, Johnson TD, Hultin P, Matud J, Detels R. Immunologic effects of combined protease inhibitor and reverse transcriptase inhibitor therapy in previously treated chronic HIV-1 infection. AIDS 1998; 12: 1833-44.
[11] Hazenberg MD, Hamann D, Schuitemaker H, Miedema F. T cell depletion in HIV-1 infection: how CD4+ T cells go out of stock. Nat Immunol 2000; 1: 285-9.

[12] Silvestri G, Feinberg MB. Turnover of lymphocytes and conceptual paradigms in HIV infection. J Clin Invest 2003; 112: 821-4.

[13] McCune JM. The dynamics of CD4+ T-cell depletion in HIV disease. Nature 2001; 410: 974-9.

[14] Sereti I, Martinez-Wilson H, Metcalf JA, et al. Long-term effects of intermittent interleukin 2 therapy in patients with HIV infection: characterization of a novel subset of $\mathrm{CD} 4(+) / \mathrm{CD} 25(+) \mathrm{T}$ cells. Blood 2002; 100: 2159-67.

[15] Hamann D, Baars PA, Hooibrink B, van Lier RW. Heterogeneity of the human CD4+ T-cell population: two distinct CD4+ T-cell subsets characterized by coexpression of CD45RA and CD45RO isoforms. Blood 1996; 88: 3513-21.

[16] Sereti I, Herpin B, Metcalf JA, et al. CD4 T cell expansions are associated with increased apoptosis rates of $\mathrm{T}$ lymphocytes during IL-2 cycles in HIV infected patients. AIDS 2001; 15: 1765-75.

[17] Trushin SA, Algeciras-Schimnich A, Vlahakis SR, et al. Glycoprotein 120 binding to CXCR4 causes p38-dependent primary $\mathrm{T}$ cell death that is facilitated by, but does not require cell-associated CD4. J Immunol 2007; 178: 4846-53.

[18] Picker LJ, Treer JR, Ferguson-Darnell B, Collins PA, Buck D, Terstappen LW. Control of lymphocyte recirculation in man. I. Differential regulation of the peripheral lymph node homing receptor $\mathrm{L}$-selectin on $\mathrm{T}$ cells during the virgin to memory cell transition. $\mathrm{J}$ Immunol 1993; 150: 1105-21.

[19] Baars PA, Maurice MM, Rep M, Hooibrink B, van Lier RA. Heterogeneity of the circulating human CD4+ T cell population. Further evidence that the CD4+CD45RA-CD27- T cell subset contains specialized primed T cells. J Immunol 1995; 154: 17-25.

[20] Prince HE, York J, Jensen ER. Phenotypic comparison of the three populations of human lymphocytes defined by CD45RO and CD45RA expression. Cell Immunol 1992; 145: 254-62.

[21] Wallace DL, Beverley PC. Phenotypic changes associated with activation of CD45RA+ and CD45RO+ T cells. Immunology 1990; 69: 460-7.

[22] Porichis F, Vlata Z, Hatzidakis G, Spandidos DA, Krambovitis E. HIV-1 gp120/V3-derived epitopes promote activation-induced cell death to superantigen-stimulated $\mathrm{CD} 4+/ \mathrm{CD} 45 \mathrm{RO}+\mathrm{T}$ cells. Immunol Lett 2007; 108: 97-102.

[23] Watts TH. TNF/TNFR family members in costimulation of $\mathrm{T}$ cell responses. Annu Rev Immunol 2005; 23: 23-68.

[24] Hendriks J, Xiao Y, Borst J. CD27 promotes survival of activated T cells and complements CD28 in generation and establishment of the effector T cell pool. J Exp Med 2003; 198: 1369-80. 